\title{
Development of stroboscopic muography
}

\author{
H. K. M. Tanaka \\ Earthquake Research Institute, the University of Tokyo, Tokyo, Japan \\ Correspondence to: H. K. M. Tanaka (ht@ @riken.jp)
}

Received: 9 July 2012 - Published in Geosci. Instrum. Method. Data Syst. Discuss.: 3 September 2012 Revised: 26 November 2012 - Accepted: 3 December 2012 - Published: 24 January 2013

\begin{abstract}
Conventional muon radiography has concentrated on non-destructive studies of stationary objects with relatively long exposure times required to achieve sufficient muon statistics. A muon detection system with real time readings and a high spatial resolution detector, enables the investigation of dynamic processes in a stroboscopic mode, where image frames are synchronized with the phases of the dynamic target. Although the natural cosmic ray muon flux is quite low for imaging a rapid process, repetitive processes can still be studied with high time resolution by integrating a large number of frames synchronized to the process. In this paper we demonstrate the stroboscopic imaging capabilities of cosmic ray muon radiography with scintillation counters and the muon readout module. The dynamics of a chemical and melting process in an electric furnace with a size of $30 \mathrm{~m}$ water equivalent in diameter was investigated as an example, and stroboscopic images were obtained for hourly changes, with acquisition frames of $400 \mathrm{~h}$ each. The results of these experiments demonstrate the future potential for muon radiography of repetitional process, such as magma flow in a conduit.
\end{abstract}

\section{Introduction}

Cosmic ray muon radiography and tomography is becoming a new technique for non-destructive studies of gigantic objects. Muon radiography was first proposed to determine the thickness of an overburden of a horizontal tunnel in the Snowy Mountains in Australia (George, 1955). He measured the muon flux inside and outside the tunnel to compare them and confirm that a reduction in the muon flux reflects the average density of the overburden. In 1969, Alvarez and others applied George's idea to the "Great Pyramid" of Giza to search for undiscovered chambers (Alvarez et al., 1970). The high penetration depth of muons in matter enables studies of the internal structure of geological objects such as volcanoes (Tanaka et al., 2007, 2008, 2009a, 2010; Lesparre et al., 2012) and seismic faults (Tanaka et al., 2011). Interactions of very high-energy cosmic rays with the atmosphere produce a flux of high-energy muons. These muons come mostly from the vertical, following a known zenith angle distribution. It is also known that muons arrive in the horizontal direction with a smaller average intensity, but with a higher intensity at energies above a few $100 \mathrm{GeV}$. These horizontal muons can be used for muon radiography of volcanoes. Muon radiographic imaging relies on attenuation contrast within the objects. If the target geometry is known, the information from counting muon events in the detector at different arrival angles can be used to infer the average density of the matter through which the muons traveled, because only the integrated effect along the travel path leads to the attenuation of the flux. The required observation time of the detection system is inversely proportional to the area of the detector used.

Most of the muon radiography measurements have been applied to stationary objects, but only a few dynamic studies have so far been performed (e.g. imaging before and after the 2009 Asama eruption; Tanaka et al., 2009b). One of the reasons which makes it difficult for us to perform real time or rapid time sequence radiography is the relatively low intensity of the cosmic ray muon flux that leads to long integration times to reach an adequate contrast in muon transmission images. Furthermore, integration times are even longer in the case of high resolution radiography. However, such low cosmic ray muon flux can be compensated for by averaging a large number of short acquisition frames, as in the case of periodic processes. After adding up of all the images for the corresponding time point, an average snapshot image with better statistics is obtained. The detection systems can be synchronized with the processes of the target, and sufficient 
muon statistics can be achieved in stroboscopic imaging, after integration of a number of frames. The image is built up by the superposition of a number of short-time datasets.

One of the potential applications for stroboscopic imaging is the investigation of recurrent processes of volcanic eruption, which for example can be seen in Stromboli Volcano in Italy or Pu'u O'o of Kilauea Volcano in Hawaii. Stromboli has been erupting every five to $20 \mathrm{~min}$ for thousands of years, with between four and six vents in the summit crater, and lava-flows are commonly emitted several times a year, cascading down on the northern flanks of the volcano (D'Auria, 2010). In Kilauea, the eruption was characterized by periodic episodes of lava fountaining and the creation of lava lakes, after massive drainbacks of the lava into the vent. Frequent overflows from a lava lake formed over the new vent have been building a broad, low shield. These drainbacks presumably kept refilling the magma chamber and allowed a longer persistence of the eruption (Garcia et al., 2000). Stroboscopic muon radiography is an approach which can reveal the eruption dynamics of such volcanoes.

Since the cosmic ray muon flux limits the required image integration time to achieve an acceptable number of muons, the resolution of stroboscopic muon radiography is only limited by the characteristics of the parameters of the detection system (detection area, temporal and spatial resolution). In this work, stroboscopic muon radiography was first performed. Data collection was based on the stroboscopic method for investigations of repetition processes of the chemical reaction in an electric carbide furnace: a set of images was recorded in exactly the same position by the electric furnace as a demonstration of stroboscopic imaging of a repetitional process inside a volcanic conduit. After adding up all the images for the corresponding phase, an average snapshot with better statistics was obtained.

\section{Experimental}

In this work, we investigated the capabilities of the detection system comprising plastic scintillator strips (Bicron BC408 ) whose slot ( $2 \mathrm{~mm}$ in depth and $2 \mathrm{~mm}$ in width) contains a wavelength-shifting (WLS) fiber with a diameter of $1 \mathrm{~mm}$ (Bicron BCF-91A) that conveys the optical signal to a multianode photomultiplier tube (MAPMT) (HAMAMATSU H8500). The size of each scintillator strip (Bicron BC-408) is $40 \mathrm{~cm}(\mathrm{~L}) \times 3.3 \mathrm{~cm}(\mathrm{~W}) \times 1.5 \mathrm{~cm}(\mathrm{H})$. The plane contains 24 plastic scintillators arranged along the $x$ and $y$ coordinates like a lattice, to determine the vertex point by determining the coincidence of two signals from two scintillator strips; the active area of the detector is $40 \times 40 \mathrm{~cm}^{2}$ at maximum geometrical acceptance. Electromagnetic (EM) showers and multi-muon events are rejected by discarding events when more than one signal from the same layer (detector plane) coincides in a time gate of $100 \mathrm{~ns}$ (multiplicity cut). The prior experiments on muon imaging with
FPGA-based (field programmable gate array) Muon Readout Module, developed by Uchida et al. (2010) demonstrated the very low readout noise, high-speed event counting, and accurate timing of the acquisition frames. The readout frames can be as short as one microsecond. The histogram of the muon events as a function of azimuth and elevation angles was generated on an internal memory of the FPGA as an HTML (hyper text markup language) file and was accessed and stored by a remote computer.

The detection system was installed next to an electric carbide furnace. The diameter of the furnace is $14 \mathrm{~m}$. The distance between the center of the furnace and the detection system is $15 \mathrm{~m}$. The distance between the two segmented scintillation detector planes (= position sensitive detector planes: PSD planes) was $100 \mathrm{~cm}$, and a root means square (RMS) angular resolution of the system was $\pm 14 \mathrm{mrad}$ at an interval of $33 \mathrm{mrad}$. The minimum resolvable distance (spatial resolution) at a target object is then defined by the minimum resolvable angle (RMS angular resolution) and the distance between the object and the detection system $(R \mathrm{~m})$ is $\pm 0.014 \pm R$ m. "Forward-directed" muon trails can be distinguished from "backward-directed" ones by choosing positive or negative angles, respectively, because muons arriving from below (neutrino-induced muons) are negligible to those arriving from above. The combination of high angular resolution, relatively large active area, and the cosmic ray muon intensity allows stroboscopic imaging with acquisition frames as short as $400 \mathrm{~h}$ with a density resolution of $13 \%$ for a target with a size of $30 \mathrm{~m}$ water equivalent (mwe) (Tanaka, 2012). The resolution of a similar type of the detection system was also verified using cosmic ray muon radiography of a large steel block (Tanaka, 2012). These images and the results of our previous calibrations of spatial resolution of the transmission images confirm that the spatial resolution is limited only by the angular resolutions and intervals of the detection system.

The position and orientation of the detection system were optimized by using 3-D CAD (computer aided design) models of the buildings and plants in the factory, which provide muon path lengths as a function of azimuth and elevation angles. The location was chosen so that the "backwarddirected" muons were not affected by the existence of other buildings and plants. Although the whole area of the detection system is sensitive to muons that come from the nearhorizontal direction, it does not catch all the muons that enter at large angles horizontally. The azimuth distribution of the "backward-directed" muons was then used to correct the geometrical acceptance of the detection system. Total weight of the detection system is about $15 \mathrm{~kg}$, and therefore it was possible for us to place the system in relatively small housing that can keep higher pressures inside, in order to avoid dust contamination (Fig. 1). The observation altitude was near the sea level.

A carbide molten layer has different densities (2.0 $2.1 \mathrm{~g} \mathrm{~cm}^{-3}$ ) from other layers (namely, heat, diffusion, and 

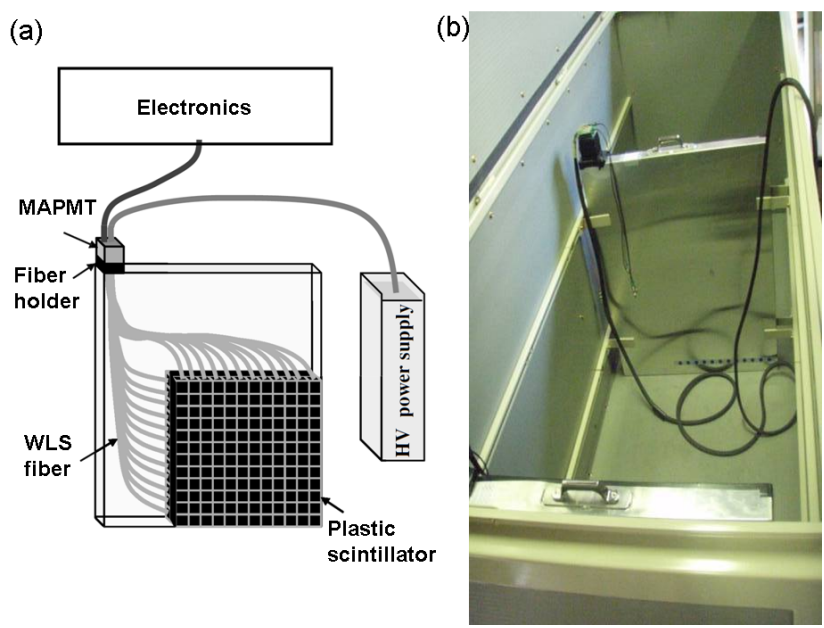

Fig. 1. Schematic view of the position sensitive detector (PSD) plane (a) and a photograph of the present experimental setup in steel housing (b). The housing was designed so that the distance between two PSD planes is adjustable, and the inside air pressure is controllable.

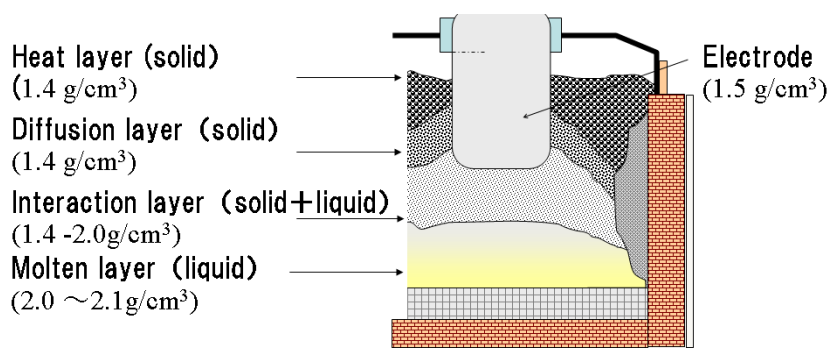

Fig. 2. Reaction model of a electric carbide furnace.

reaction layers) $\left(1.4-2.0 \mathrm{~g} \mathrm{~cm}^{-3}\right)$ in the furnace (Fig. 2). The thickest part of the furnace is therefore $\sim 30$ mwe. Based on the steel block experiment, it was confirmed that a density resolution of $13 \%$ for total data acquisition time of $400 \mathrm{~h}$ is sufficient for us to image the boundary between the melting and other layers in the furnace. Since electric furnaces require a large amount of electricity, electric load patterns change according to the electricity price per $\mathrm{kWh}$. The plant operational conditions, therefore, frequently change in a day. Conventionally, the internal conditions of the furnace have been estimated based on the operator's experiences and estimation imagination. We attempted to obtain stroboscopic images with better statistics by adding up all the snapshots for the corresponding electric load patterns. Periodic electric load patterns are shown in Fig. 3. The load is a purely twostate system, where there is a certain rise time, but the fall time is negligible.

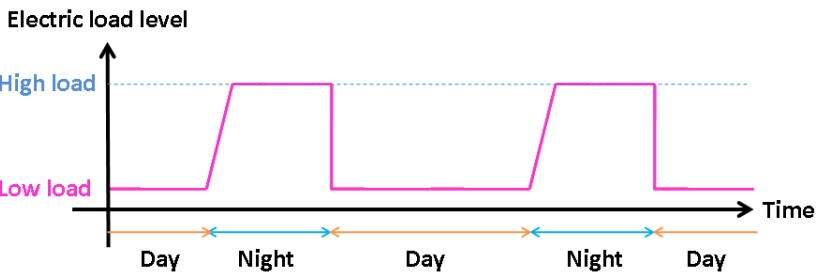

Fig. 3. Periodic electric load pattern applied to the electric furnace.
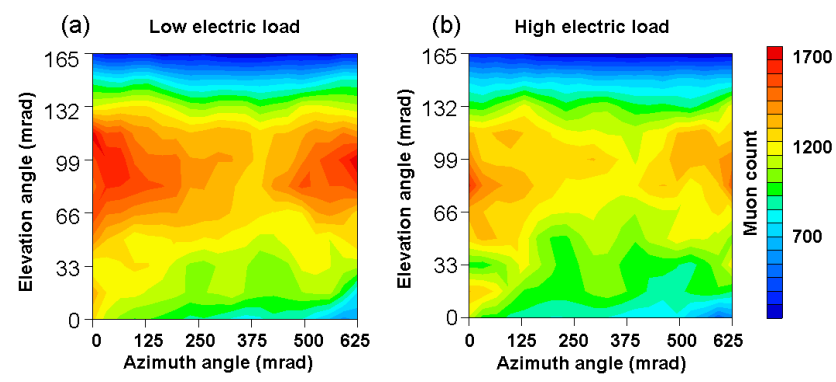

Fig. 4. Stroboscopic muographs reconstructed with acquisition frames of $400 \mathrm{~h}$ each to achieve the high spatial resolution of the electric furnace under (a) low electric load and (b) high electric load conditions.

\section{Results and discussions}

The low muon statistics in the dynamic muon radiography can be substantially improved if the process under study is repetitional. In this case, a number of phase-locked frames can be integrated to form one image, which has sufficient statistics without degrading spatial resolution of the image. As proof of principle measurement, the electric furnace under the different load patterns has been imaged in a stroboscopic mode with 10-min frames, but total data acquisition time is $400 \mathrm{~h}$ per image in this work. The total numbers of collected muons are 226694 for a high-electric load condition (total observation time of $195 \mathrm{~h}$ ), and 238137 for a lowelectric load condition (total observation time of $205 \mathrm{~h}$ ), respectively. The acquisition frames are synchronized to the trigger provided by the electric load levels. A number of frames needs to be integrated in order to achieve the high spatial resolution of the resulting "frozen in time" image.

Figure 4 compares muographs between high- and lowelectric loads. This figure shows the muon transmission rate distribution projected on the cross-sectional plane that is parallel to the detector plane. The more materials, the fewer the muons there will be that have enough energy to get through the furnace. There, the "more materials" simply refers to higher density in the vertical direction because of the cylindrical shape of the target. When we perform muon radiography of a target with such a simple geometry, and when the information on absolute density values is not necessary, muon transmission mappings are not usually converted into average density mappings in order to reduce errors associated with 
3-D geometrical modeling of the experimental setup that includes the target and the detection system. Therefore, only the horizontal distribution of the muons was corrected in this image. In Fig. 4, we can see an increase in the muon transmission rate towards the edge of the furnace in general, and a maximum transmission rate at an elevation angle of $165 \mathrm{mrad}$ ( $2.5 \mathrm{~m}$ above the base at the center of the furnace), which comes from a competition between a decrease in geometrical acceptance and an increase in zenith-angular dependence of the muon intensity.

Green to blue regions in the figure show relatively low muon transmission rates that indicate high densities. When higher electric loads are applied, a thickness of the high density region increases. This is consistent with our vision that temperature rises and more molten materials are deposited at the bottom of the furnace when higher electric loads are applied. Overall, our detection system reached the acceptable levels for the stroboscopic imaging of the target, the size of which is that of an industrial plant.

\section{Conclusions}

With conventional muon radiography, only averaged flow distributions can be studied. The example of muon imaging of dynamic processes shown in this work indicate that a muon detection system using scintillation counters and the muon readout module can provide some novel, stroboscopic imaging techniques. A number of frames must be acquired since the muon statistics are limited by the natural cosmic ray flux. However, even with very limited muon statistics, dynamic processes such as magma propagation, or twophase flow in a conduit, can be studied with short acquisition frames by using a detection system with a larger active area and integration over a large number of frames.

For the case study of the Stromboli eruption, here we assume a muon detector with a size of $40000 \mathrm{~cm}^{2}$, located at a distance of $200 \mathrm{~m}$ from the vent, with a radius of $10 \mathrm{~m}$ to detect it through rock $\left(2 \mathrm{~g} \mathrm{cc}^{-1}\right)$, with a thickness of $200 \mathrm{~m}$. The penetrating muon flux for an elevation of $15^{\circ}$ is $5 \times 10^{-5} \mathrm{sr}^{-1} \mathrm{~cm}^{-2} \mathrm{~s}^{-1}$ if the vent is completely filled, and $7 \times 10^{-5} \mathrm{sr}^{-1} \mathrm{~cm}^{-2} \mathrm{~s}^{-1}$ if it is vacant. The actual muon flux will be in between these numbers. The velocity and mass of pyroclast will be monitored so that stroboscopic muography can be synchronized to the eruption events. Taddeucci et al. (2012) applied high speed video imaging to monitoring the active vents of Stromboli volcano and reported that the pyroclast ejection velocity decays within one second for each explosion event. We therefore set the time resolution of muography to be $1 \mathrm{~s}$. With the assumed active area of the detector and the required angular resolution $(100 \mathrm{mrad})$ to resolve the vent at a distance of $200 \mathrm{~m}$, the number of muons we can collect is 0.02 and $0.03 \mathrm{~s}^{-1}$ for the filled and vacant vent, respectively. We therefore need 2500 eruption events to separate them at $3 \sigma$ confidence level. Observing
2500 eruption events is not unrealistic if we consider that 110 eruptions were observed in Stromboli between 14 and 17 October 2007 (Goto et al., 2008).

Lava flows erupting from Pu'u O'o are typically 5-10 m thick and at speeds of $50-500 \mathrm{~m} \mathrm{~h}^{-1}$. As in the case of monitoring Pu'u O'o eruptions, we assumed a simple cylindrical model with a diameter of $500 \mathrm{mwe}$, and a uniform density of $2.0 \mathrm{~g} \mathrm{~cm}^{-3}$ that includes a magma pathway $20 \mathrm{~m}$ in diameter, then calculated the time required to detect magma in the pathway. The number of muons that can cross 500 and 460 mwe is 0.15 and $0.18 \mathrm{~m}^{-2} \mathrm{~min}^{-1}$, respectively. If we assume that the detector's active area and angular resolution is $1 \mathrm{~m}^{2}$ and $100 \mathrm{mrad}$, and 50 frames are available for the integration, the time required to detect the magma at $2 \sigma$ confidence level will be $20 \mathrm{~min}$. From 1983 to 1986 , a series of 44 lava fountains were built at Pu'u O'o, and thus, the number of frames we assumed here is reasonable.

Acknowledgements. The experiment was supported by collaborative research between DENKA (Denki Kagaku Kogyo Kabushiki Kaisha) and the University of Tokyo. The manuscript greatly benefited from careful review by Justin Albert and anonymous reviewers.

Edited by: J. Albert

\section{References}

Alvarez, L. W., Anderson, J. A., Bedwei, F. E., Burkhard, J., Fakhry, A., Girgis, A., Goneid, A., Hassan, F., Iverson, D., Lynch, G., Miligy, Z., Mousaa, A. H., Sharkawi, M., and Yazolinio, L.: Search for hidden chambers in the pyramids, Science, 167, 832839, 1970.

D'Auria, L.: Perspectives of muon radiography for Stromboli, International Workshop on Muon Radiography of Volcanoes, 1112 October 2010, Naples, Italy, 2010.

Garcia, M. O., Pietruszka, A. J., Rhodes, J. M., and Swanson, K.: Magmatic Processes During the Prolonged Pu'u 'O'o Eruption of Kilauea Volcano, Hawaii, J. Petrol., 967-990, doi:10.1093/petrology/41.7.967, 2000.

George, E. P.: Cosmic rays measure overburden of tunnel, Commonwealth Engineer, 455-457, 1955.

Goto, A., Ripepe, M., and Lacanna, G.: Analysis of video images and high rate sampled acoustic waves on Stromboli volcano, Abstracts Volcanological Society of Japan 70, 2008-10-10, 2008.

Lesparre, N., Gibert, D., Marteau, J., Komorowski J., Nicolin, F., and Coutant, O.: Density muon radiography of La Soufrière of Guadeloupe volcano: comparison with geological, electrical resistivity and gravity data, Geophys. J. Int., 185, 1-14, doi:10.1111/j.1365-246X.2012.05546.x, 2012.

Taddeucci, J., Alatorre-Ibargüengoitia, M. A., Moroni, M., Tornetta, L., Capponi, A., Scarlato, P., Dingwell, D. B., and De Rita, D.: Physical parameterization of Strombolian eruptions via experimentally-validated modeling of high-speed observations, Geophys. Res. Lett., 39, L16306, doi:10.1029/2012GL052772, 2012. 
Tanaka, H. K. M.: Evaluation of positioning and density profiling accuracy of muon radiography by utilizing a 15-ton steel block, Geosci. Instrum. Method. Data Syst. Discuss., 2, 643-656, doi:10.5194/gid-2-643-2012, 2012.

Tanaka, H. K. M., Nakano, T., Takahashi, S., Yoshida, J., Takeo, M., Oikawa, J., Ohminato, T., Aoki, Y., Koyama, E., Tsuji, H., and Niwa, K.: High resolution imaging in the inhomogeneous crust with cosmic ray muon radiography: The density structure below the volcanic crater floor of Mt. Asama, Japan, Earth Planet. Sc. Lett., 263, 104-113, 2007.

Tanaka, H. K. M., Nakano, T., Takahashi, S., Yoshida, J., Takeo, M., Oikawa, J., Ohminato, T., Aoki, Y., Koyama, E., Tsuji, H., Ohshima, H., Maekawa, T., Watanabe, H., and Niwa, K.: Radiographic imaging below a volcanic crater floor with cosmic-ray muons, Am. J. Sci., 308, 843-850, 2008.

Tanaka, H. K. M., Uchida, T., Tanaka, M., Shinohara, H., and Taira, H.: Cosmic-ray muon imaging of magma in a conduit: Degassing process of Satsuma-Iwojima Volcano, Japan, Geophys. Res. Lett., 36, L01304, doi:10.1029/2008GL036451, 2009a.
Tanaka, H. K. M., Uchida, T., Tanaka, M., Takeo, M., Oikawa, J., Ohminato, T., Aoki, Y., Koyama, E., and Tsuji, H.: Detecting a mass change inside a volcano by cosmic-ray muon radiography (muography): First results from measurements at Asama volcano, Japan, Geophys. Res. Lett., 36, L17302, doi:10.1029/2009GL039448, 2009b.

Tanaka, H. K. M., Uchida, T., Tanaka, M., Shinohara, H., and Taira, H.: Deveelopment of a portable assembly-type cosmic-ray muon module for measuring the density structure of a column of magma, Earth, Planets Space, 62, 119-130, 2010.

Tanaka, H. K. M., Miyajima, H., Kusagaya, T., Taketa, A., Uchida, T., and Tanaka, M.: Cosmic muon imaging of hidden seismic fault zones: Rainwater permeation into the mechanical fractured zones in Itoigawa-Shizuoka Tectonic Line, Japan, Earth Planet. Sc. Lett., 306, 156-162, 2011.

Uchida, T., Tanaka, H. K. M., and Tanaka, M.: Development of a muon radiographic imaging electronic board system for a stable solar power operation, Earth Planet. Space, 62, 167-172, 2010. 